Received 21 January 1997; accepted 14 July 1997.

Efrain M. Ribot, Frederick D. Quinn, Xianhe Bai ${ }^{1}$ and James J. Murtagh, Jr. ${ }^{1}$

Centers for Disease Control and Prevention

Atlanta

${ }^{1}$ Atlanta Veterans Administration Medical Center

Decatur, GA, USA

\section{Comparative PCR: An Improved Method to De- tect Gene Amplification}

BioTechniques 24:22-26 (January 1998)

With the rapid advances in tumor genetics, an increasing number of amplifications have been identified in various human neoplasms (4). Examples of oncogenes amplified in tumors include $\mathrm{N}-m y c$ and HER-2/neu, which have been proposed as prognostic markers in neuroblastoma and breast cancer, respectively $(5,7)$. To date, most of the information on the amplification of genes has been gathered from conventional Southern blot analysis, but this approach requires relatively large amounts of genomic DNA $(5-10 \mu \mathrm{g})$ and lacks sufficient sensitivity. Alternatively, comparative genomic hybridization has been used to detect and localize extended amplification domains in human cancers (3). This method, however, is not tailored to the analysis of single loci.

Recently, quantitative polymerase chain reaction (PCR) application has gained increasing importance for the evaluation of oncogene amplification. Several modifications of this approach have been described, all of them bearing significant limitations. One of the widely used methods, called differential PCR, requires amplification of both a single-copy control gene and the gene of interest in the same vial (1). This in turn requires PCR conditions that are appropriate for both primer pairs in the same reaction. In addition, it remains unclear how an interaction of the two primers interferes with the amplification. To overcome these limitations, a method termed competitive PCR makes use of artificial internal standards that share the primer-binding sites with the gene of interest (6). These standards, which are termed competitors, have to be synthesized and quantified for each gene separately.

According to most protocols, the determination of the gene amplification level also involves scintillation counting. We present a simple and rapid PCR scheme to assess gene amplification while avoiding time-intensive synthesis and use of competitors.

The method, which we termed comparative PCR, requires PCR amplification of a given gene in tumor DNA and normal DNA (most conveniently peripheral blood DNA). Because gene amplification is a hallmark of tumor DNA and is rarely found in normal hu-

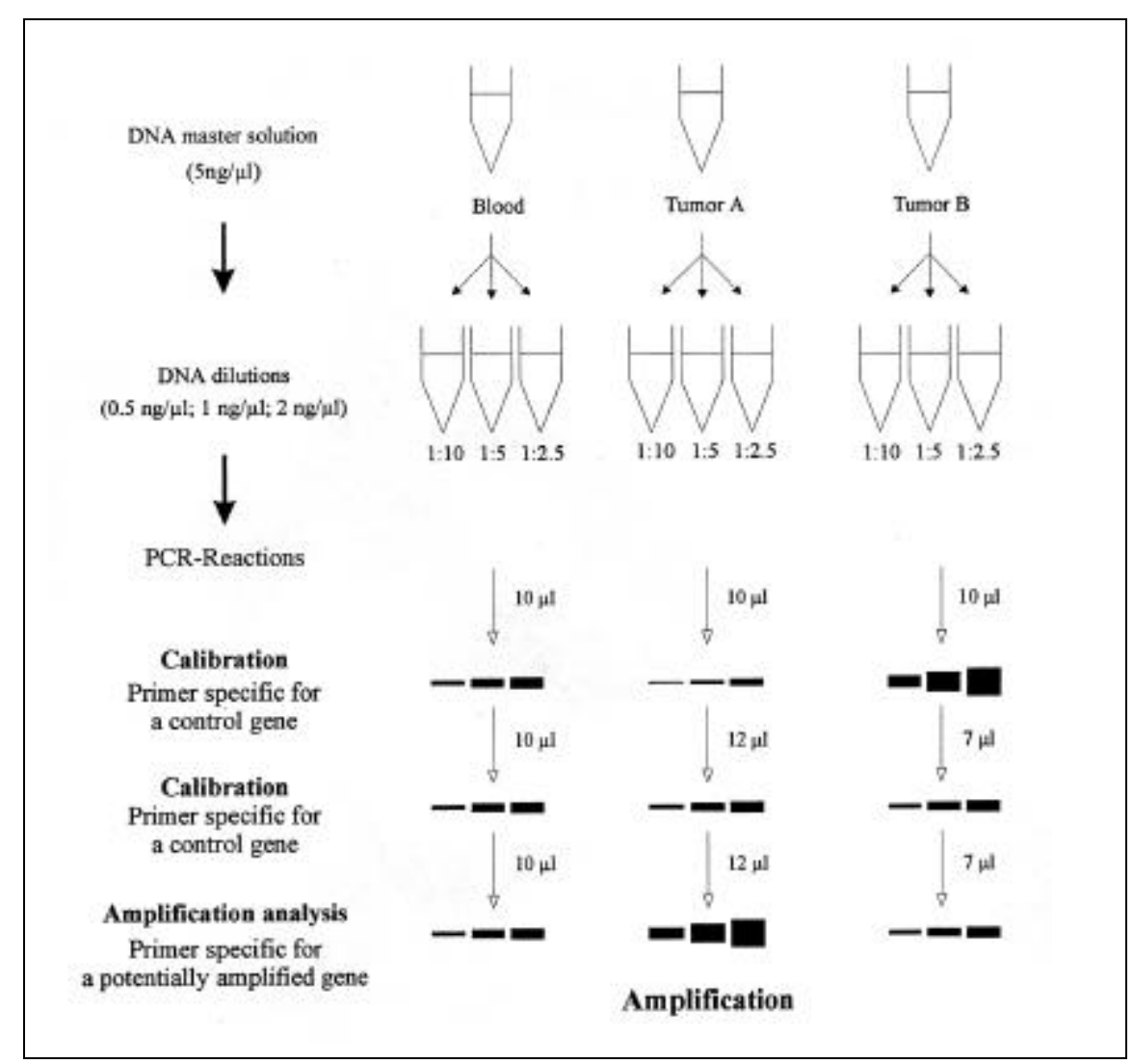

Figure 1. Reaction scheme of the comparative PCR. man cells, it is not necessary to use a sample of blood DNA from each patient (9). The signal intensity of the PCR products reflects the copy number of the gene in the tumor and peripheral blood DNA. By comparing the signals in tumor and normal DNA, the presence of gene amplification can be detected.

A necessary prerequisite for direct stained agarose gel is the calibration of the system with a primer pair specific for a control gene not amplified in tumor DNA. Furthermore, the PCR amplification is to be performed on at least three different amounts of tumor and blood DNA. The implications of this step are threefold: $(i)$ the three independent amplifications facilitate the assessment of the amplification status; (ii) they provide an internal control for the reproducibility of the experiment; and (iii) they serve as control to keep the PCR amplification within the exponential phase. A schematic representation of the experimental approach is given in Figure 1. Details to this fast and comparison of the band intensities in a 
highly efficient protocol are as follows.

PCR primers are designed specifically for the genes that are to be tested for amplification and for the sequence that serves as single-copy control. All PCR products should be in the same size range and should not exceed a length of 250 nucleotides. Specific PCR products without any additional side products are a necessary prerequisite for quantification by PCR. Unspecific products are likely to interfere with the signal intensity of the specific PCR products and therefore complicate the evaluation. As for other PCR approaches, the PCR optimization is done on peripheral blood DNA by varying magnesium chloride concentration, annealing temperature and the amount of DNA template.

As mentioned above, three different dilutions of template DNA are amplified by PCR. In our hands, dilutions of $0.5,1$ and $2 \mathrm{ng} / \mu \mathrm{L}$, which are prepared from a master solution of $5 \mathrm{ng} / \mu \mathrm{L}$, were best suited. To reflect the amount of starting material, the PCR must be kept within the exponential phase. To this end, the number of cycles is reduced to a level that still yields PCR products visible on agarose gels while using the lowest concentration of template DNA. To increase the sensitivity of the DNA detection, the DNA can be stained with SYBR ${ }^{\circledR}$ Green I (Molecular Probes, Eugene, OR, USA), which is $5-10$ times more sensitive than ethidium bromide.

The tumor DNA is diluted the same way as the peripheral blood DNA. It is crucial that sufficient amounts of each dilution be made prior to the experiment. Even minor variations in concentration will result in dramatic changes in amounts of PCR products. The required amount of tumor DNA depends on the number of genes tested for amplification. In our hands, approximately $70 \mathrm{ng}$ tumor DNA are needed for calibration purposes with the control gene. Importantly, a single control can be used for a large number of candidate genes. The analysis of the candidate genes required $35 \mathrm{ng}$ tumor DNA per gene, making it possible to analyze, for example, five candidate genes with roughly $250 \mathrm{ng}$ DNA. The minimal amount of DNA required even makes it possible to analyze very small tumor biopsies. In our studies, we analyzed DNA out of tumor samples from routine diagnostic endoscopies. The yield of DNA varied between $200 \mathrm{ng}$ and 5 $\mu \mathrm{g}$ tumor DNA per sample. Furthermore, we demonstrated that DNA from 1-2 slides of paraffin-embedded tumor sections is sufficient for analyzing the amplification status of one potentially amplified gene. As for the time consideration, two calibration steps are usually required to adjust tumor and control DNA, and a single step is necessary to determine the amplification status. Using this time-saving PCR approach, it is possible to calibrate many DNA samples in less than two days and to analyze the status of a potentially amplified gene in less than one day.

Before determining the amplification level of the candidate gene, it is necessary to compare the PCR products of the single-copy control in tumor vs. blood DNA. Although the concentrations of the DNA master solutions have been determined by optical densitomet-

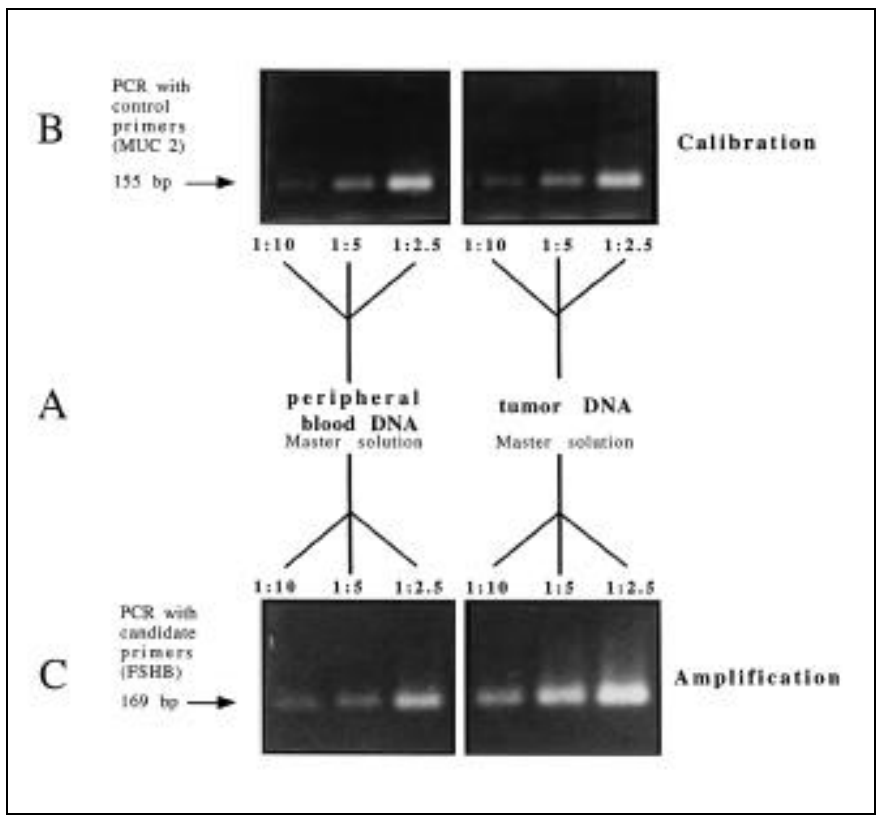

Figure 2. Flow diagram of the comparative PCR method. (A) Dilution of DNA master solutions $(5 \mathrm{ng} / \mu \mathrm{L})$ of peripheral blood DNA and tumor DNA. (B) Calibration of DNA concentrations using a primer pair specific for the $M U C 2$ gene as control (8). (C) Analysis of the FSHB gene as an example for an amplified gene (2). Both PCR amplifications were carried out using a step program $\left(94^{\circ} \mathrm{C}\right.$ for $1 \mathrm{~min}, 58^{\circ} \mathrm{C}$ for $45 \mathrm{~s}[M U C 2] / 57^{\circ} \mathrm{C}$ for $45 \mathrm{~s}[F S H B]$ and $72^{\circ} \mathrm{C}$ for 45 $\mathrm{s} ; 26$ cycles) and an $\mathrm{MgCl}_{2}$ concentration of $1.5 \mathrm{mM}$. The primer sequences were as follows: MUC2-A: CAT TCT CAA CGA CAA CCC CT; MUC2-Z: GCA AGA GAT GTT ACT GCC C; FSHBfor: CAG TTT CAC CTC GCC TTT TC; FSHBrev: TTT CCA CAG CCC CAA ATA C.

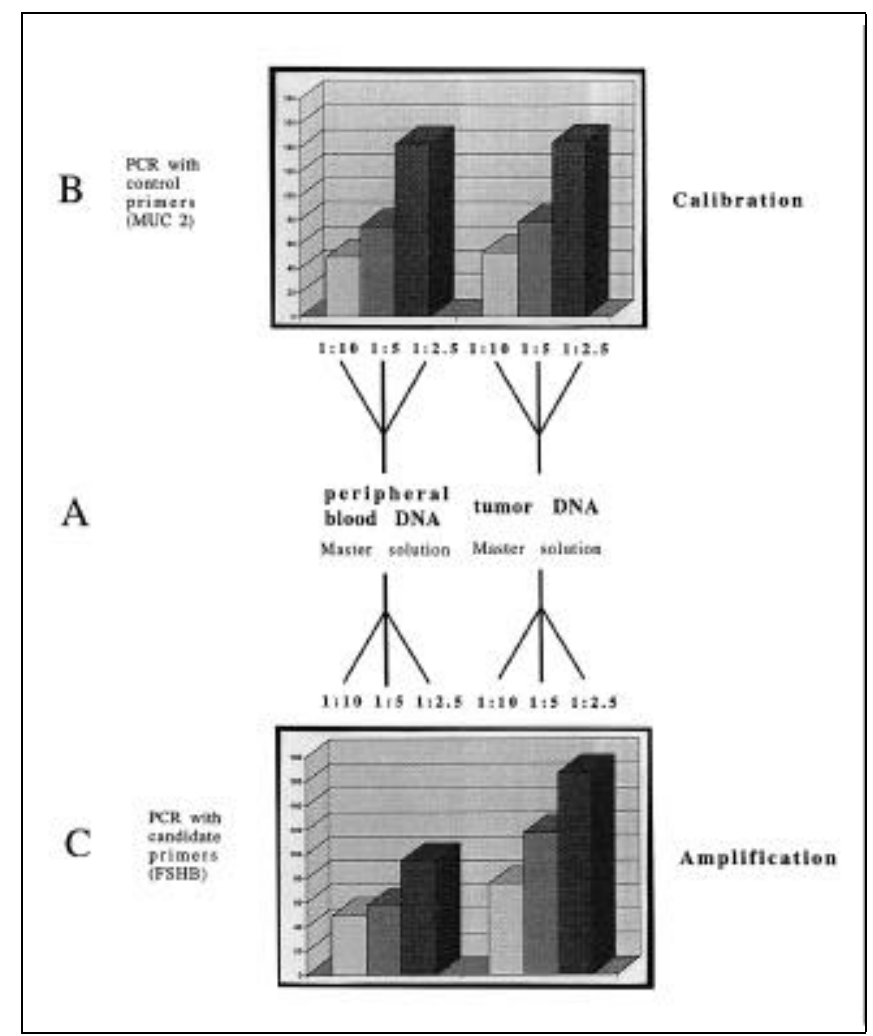

Figure 3. Densitometric evaluation of the PCR signals shown in Figure 2. The densitometric measurement of signal intensities was performed using the program NIH Image Version 1.61. The presentation is according to Figure 2. 
ric measurement, we sometimes obtained inconsistent results after the first calibration step because of an inaccurate measurement in a low concentration range. In case there are variations in signal intensity between blood and tumor DNAs, the amount of the PCR templates can be adjusted accordingly (Figure 2B). In addition to simple comparison of the signal intensities in the agarose gel, a densitometric measurement is possible (Figure 3B). However, in general, only minor adjustments are necessary in our experience.

We used $10 \mu \mathrm{L}$ of each dilution for the first calibration PCR, which was quantitatively separated by gel electrophoresis. It is convenient to include several tumor DNAs, provided they can be run on the same gel as the control.

To identify DNA amplifications, PCRs are carried out with calibrated amounts of DNA. After gel electrophoresis and staining of the PCR products, amplifications are indicated by intense signals in tumor DNA in comparison to weaker signals in peripheral blood DNA. An example of a DNA amplification identified by this approach is shown in Figures 2C and 3C. As demonstrated, the signal intensities of all three DNA dilutions are stronger in the tumor DNA than in the control DNA. This serves as an internal control to ensure that the stronger signals do represent amplifications and do not result from differences in PCR efficiency or pipetting errors. If the results are not consistent for all dilutions, the experiment is to be discounted.

Recently, we used competitive PCR to analyze an extended region of gene amplification on chromosome 11q14. The results of the competitive PCR experiment were reproduced by our novel method but within significantly shorter time and with less effort. So far, we have tested more than 100 DNA samples of different tumor types for amplification using comparative PCR. The results of these experiments are in accordance with both in situ hybridization and Southern blot hybridization data. In summary, comparative PCR is an extremely reliable technique that has already been routinely used in several studies on DNA amplification.

Unlike comparable protocols such as competitive PCR, our novel method combines increased flexibility, time efficiency, reduced costs and high reproducibility.

\section{REFERENCES}

1.Frye, R.A., C.C. Benz and E. Liu. 1989. Detection of amplified oncogenes by differential polymerase chain reaction. Oncogene 4:11531157.

2.Jameson, J.L., C.B. Becker, C.M. Lindell and J.F. Habener. 1988. Human follicle-stimulating hormone beta-subunit gene encodes multiple messenger ribonucleic acids. Mol. Endocrinol. 2:806-815.

3.Pinkel, D., J. Landegent, C. Collins, J. Fuscol, R. Segraves, J. Lucas and J. Gray. 1988. Fluorescence in situ hybridization with human chromosome-specific libraries: detection of trisomy 21 and translocations of chromosome 4. Proc. Natl. Acad. Sci. USA 85:9138-9142.

4.Schimke, R.T. 1990. The search for early genetic events in tumorgenesis: an amplification paradigm. Cancer Cells 2:149-151.

5.Seeger, R.C., G.M. Brodeur, H. Sather, A. Dalton, S.E. Siegel, K.Y. Wong and D. Hammon. 1985. Association of multiple copies of the N-myc oncogene with rapid progression of neuroblastomas. N. Engl. J. Med. 313:1111-1116.

6.Siebert, P.D. and J.W. Larrick. 1992. Competitive PCR. Nature 359:557-558.

7.Slamon, D.J., W. Godolphin, L.A. Jones, J.A. Holt, S.G. Wong, D.E. Keith, W.J. Levin, S.G. Stuart et al. 1989. Studies of the HER-2/neu proto-oncogene in human breast and ovarian cancer. Science 244:707-712.

8.Smith, M.W., S.P. Clark, J.S. Hutchinson, Y.H. Wei, A.C. Churukian, L.B. Daniels, K.L. Diggle, M.W. Gen et al. 1993. A sequence-tagged site map of human chromosome 11. Genomics 17:699-725.

9.Wright, J.A., H.S. Smith, F.M. Watt, M.C. Hancock, D.L. Hudson and G.R. Stark. 1990. DNA amplification is rare in normal human cells. Proc. Natl. Acad. Sci. USA 87:1791-1795.

This work was supported by a grant from the Deutsche Forschungsgemeinschaft (SFB 399 Project A1). Address correspondence to Dr. Eckart U. Meese, Department of Human Genetics, Medical School, University of Saarland, Bau 60, 66421 Homburg/Saar, Germany. Internet: hgemee@ med-rz.uni-sb.de

Received 26 August 1996; accepted 8 July 1997.

\section{Nicole Brass, Dirk Heckel and Eckart Meese \\ University of Saar \\ Homburg/Saar, Germany}

\section{Adaptor PCR for the Specific Amplification of Unknown DNA Fragments}

BioTechniques 24:26-28 (January 1998)

Several polymerase chain reaction (PCR) methods have been described to amplify unknown DNA fragments adjacent to known DNA regions using only one specific primer. All these methods use adaptors or tailing to add a known DNA sequence to a restriction site of the unknown DNA. The most applied PCR-based method has been developed by Arnold and Hodgson using vectorettes (1). Other methods using a similar approach are designated as anchored PCR (3) or rapid amplification of cDNA ends (RACE; Reference 2). Inverse PCR $(4,6,7)$ using two specific primers at both ends of a known sequence with direction to the unknown part of the DNA depends on circularization of the fragment of interest. Circularization is the most crucial step in inverse PCR, especially when used to specifically amplify fragments out of a complex mixture of restriction fragments. To enhance specificity of the above-mentioned methods, PCR products from the first round of PCR need to be re-amplified with nested primers.

We have developed a method to specifically amplify unknown DNA fragments out of a complex mixture of genomic DNA without re-amplifying. This approach is based on singlespecific-primer PCR (SSP-PCR) (5) and degradation of double-stranded (ds)DNA and has been used to identify NotI-linking clones in physical mapping of the Coxiella burnetii chromosome.

For this purpose, $C$. burnetii total DNA has been shotgun-cloned as NotI/ EcoRI fragments and sequenced. Contiguous $N o t \mathrm{I} / E \operatorname{coRI}$ fragments were identified by PCR using primers derived from the sequences of the fragments and directed to the NotI restriction site.

Fragments that could not be amplified that way (i.e., missing fragments adjacent to known NotI/EcoRI frag- 